\title{
Polymorphism within molecular systems revelaed by 3D electron diffraction
}

\section{Edward Thomas Broadhurst}

\author{
The University of Edinburgh, Edinburgh, United Kingdom; \\ s1371070@ed.ac.uk
}

Following the in-situ development of glycine polymorphs from an aqueous solution via 3D electron diffraction, revealing three polymorphs crystallizing at differing timepoints according to stability. Beta glycine forms after 3 minutes, followed by alpha glycine after only one minute more. Gamma glycine forms after prolonged standing. The same methodology was applied to carbamazepine which, alongside the expected dihydrate form, shows four forms after 30 seconds of crystallization. When the time is reduced to 20 seconds, dark, liquid-like, droplets appear to agglomerate together and form the dihydrate. This suggests the dihydrate forms via the non-classical nucleation similar to liquid-liquid phase separation. High pressure X-ray crystallography is a well-established technique for studying polymorphism and was used to probe the nature of the dimer interactions within isostructural organic 'Blatter' radicals. One of which showed a pseudo $2^{\text {nd }}$ order phase transition. This transition is driven by flexibility on the phenyl ring which rotates and allows the benzotriazine moiety to flatten and become more planar. Implementation of 3DED/MicroED technique on TEM in Edinburgh for routine structure solution, previously only used for imaging has also been successfully accomplished.

Broadhurst, E. T., Xu, H., Clabbers, M. T., Lightowler, M., Nudelman, F., Zou, X., \& Parsons, S. "Polymorph evolution during crystal growth studied by 3D electron diffraction" (2020), IUCrJ, 7(1), 5-9Keywords: Electron diffraction, dynamical diffraction, 3DED, D-LACBED

Keywords: 3D electron diffraction, crystallization, polymorphism, nucleation 